FACULDADE DE CIÊNCIAS ECONÔMICAS DA UFRGS
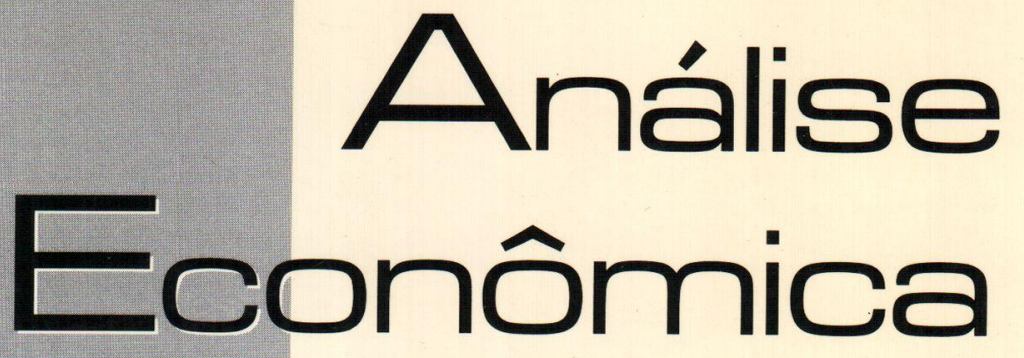

A CIÉNCIA ECONOMICA NA EUROPA E NOS Estados Unidos: PluRAlismo Versus MONISMO

ANTONio Maria da SiLveira

A EXPANSÃO DO COMERCIO MUNDIAL: IMPLICACOO ES SOBRE A HIPÓ TESE DE CONVERGENCIA

MANOEL BOSCO DE ALMEIDA

O SETOR AGRICOLA NA ÁREA DE LIVRE COMERCIO DAS AMÉRICAS: DESAFIOS DA INTEGRAÇÃO REGIONAL

PAULO D. WAQUIL.

PEQUENOS E MEDIOS FABRICANTES DE BENS DE CAPITAL FRENTE AS MUDANÇAS NA ECONOMIA BRASILEIRA

HOYEDO NUNES LINS

RISCOS NA ATIVIDADE BANCÁRIA EM CONTEXTO DE ESTABILIDADE DE PREÇOSE DE ALTA INFLAÇÃO

LUIS FERNANDO RODRIGUES DE Paula

O FIO DA NAVALHA DE HARROd EA RESPOSTA DA ESCOLA DE CAMBRIDGE MARIo AUGusto Bertella

A DINAMMICA FINANCEIRA INTERNACIONAL E A TENDENCIA Ã DOLARIZAÇÄO DAS ECONOMIAS LATINO-AMERICANAS

MARCOS ANTONIO MACEDO CinTRA

TEMPORALIDADE DA RIQUEZA - TEORIA DA DINAMMICA E FINANCEIRIZAÇÃO DO CAPITALISMO - RESENHA

Gentil Corazza

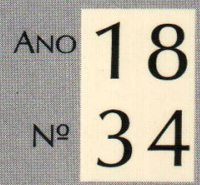


Universtode Federal do Rio Grande do Sul

Reitora: Prof ${ }^{a}$. Wrana Maria Panizzi

Faculdade de Crências Econômicas.

Diretora: Prof ${ }^{4}$. Otilia Beatriz Kroeff Carrion

Centro de Estudos e Pesquisas Econômicas

Diretor: Prof. Fernando Ferrari Filho

Departamento de Ciências Económicas

Chefe: Prof. Luiz Alberto Oliveira Ribeiro de Miranda

Departamento de Crências Contábeis e Atuariais

Chefe: Prof. João Marcos Leão da Rocha

Curso de Pós-Graduação em Economia

Coordenador: Prof Marcelo Savino Portugal

Curso de Pós-Graduação em Desenvolvimento Rurai

Coordenador: Prof Carlos Guilherme A. Mielitz Netto

Consel.ho Eomorial: Achyles B. Costa, Aray M. Feldens, Carlos A Crusius, Carlos G. A Mielitz Netto, Eduardo A. Maldonado Filho, Eduardo P. Ribeiro, Eugênio Lagemann, Fernando Ferrari Filho, Gentil Corazza, Jan A. Kregel, Marcelo S. Portugal, Nali J. Souza, Otília B. K Carrion, Paulo A. Spohr, Paulo D. Waquil, Pedro C. D. Fonseca, Philip Arestis, Roberto C. Moraes, Ronald Otto Hillbrecht, Stefano Florissi, Eleutério F. S. Prado (USP), Fernando H Barbosa (FGV/RJ), Gustavo Franco (PUC/RJ), João R. Sanson (UFSC), Joaquim P. Andrade (UnB), Juan H. Moldau (USP), Paul Davidson (Univ of Tennessee), Werner Baer (Univ. of Mlinois)

Comissāo Eoitonial: Eduardo Augusto Maldonado Filho, Fernando Ferrari Filho, Gentil Corazza, Marcelo Savino Portugal, Paulo Dabdab Waquil, Roberto Camps Moraes.

EdITor: Gentil Corazza

EdrTor Adjunto: Pedro Silveira Bandeira

Secretarus: Márcio Souza de Vargas

REVISĩo de teXtos: Vanete Ricacheski

Fundador: Prof. Antônio Carlos Santos Rosa

Os materiais publicados na revista Análise Econômica são da exclusiva responsabilidade dos autores. É permitida a reprodução total ou parcial dos trabalhos, desde que seja citada a fonte. Aceita-se permuta com revistas congêneres. Aceitam-se, também, livros para divulgação, elaboração de resenhas e recensões. Toda correspondência, material para publicação (vide normas na terceira capa), assinaturas e permutas devem ser dirigidos ao seguinte destinatário:

Prof. Genth Corazza

Revisia Anáxise Econômica - Av. João Pessoa, 52 CEP 90040-000 PORTO ALEGRE - RS, BRASIL

Telefones: (051) 316-3513 316-3440 - Fax: (051) 316-3990 rae@vortex.ufrgs.br

Análise Econômica

Ano $18, n^{\circ} 34$, setembro, 2000 - Porto Alegre

Faculdade de Ciências Econômicas, UFRGS, 2000

Periodicidade semestral, março e setembro

1. Teoria Econômica - Desenvolvimento Regional Economia Agrícola - Pesquisa Teórica e Aplicada -

Periódicos. I Brasil

Faculdade de Ciências Econômicas,

Universidade Federal do Rio Grande do Sul.

CDD 330.05

CDU $33(81)(05)$ 


\section{O fio da navalha de Harrod e a resposta da escola de Cambridge}

Mário Augusto Bertella ${ }^{l}$

Resumo: Este artigo procura discutir o modelo de crescimento de Harrod enquanto fonte de motivação para o aparecimento dos modelos de crescimento e distribuição pós-keynesianos de primeira geração (as teorias de Robinson e Kaldor), como também estudar estes modelos diante do que ficou conhecido na literatura como o fio da navalha de Harrod. Após analisarmos inicialmente o modelo de Harrod, nas seções seguintes, estudamos os modelos de Robinson e Kaldor, respectivamente. Ambos os autores atacam Harrod por sua falha em lidar explicitamente com a taxa de lucro e a distribuição de renda. $\mathrm{O}$ fundamento destas críticas baseia-se na rejeição do fio da navalha, ou seja, que há apenas uma única taxa garantida. A resposta dada por Robinson e Kaldor foi reconhecer que existem diferentes taxas de poupança associadas a diferentes classes sociais, flexibilizando a taxa de poupança agregada. O problema da taxa garantida, assim, desapareceria. Na seção final, fazemos uma apresentação concisa dos pontos comuns e divergentes das abordagens de Robinson e Kaldor.

Palavras-chave: Crescimento, distribuição, Harrod.

Abstract: This paper attempts to discuss Harrod's growth model as an inspiration for the appearance of the first generation post-keynesian growth and income distribution models (basically Robinson and Kaldor theories) and also study these models in the light of what was known in the literature as a Harrod's knife edge. After analysing Harrod's model, in the following sections we study Robinson's and Kaldor's models, respectively. Both criticize Harrod's theory due to his failure in dealing explicitly with profit rate and income distribution. These criticisms are based on the rejection of the knife edge, i. e., that there was only one warranted rate. Robinson's and Kaldor's answer was that existed several saving rates related to diferent social classes. Thus, the problem of a single warranted rate vanished. In the final section, a short comparison of Robinson's and Kaldor's ideas is discussed.

Key-words: Growth, distribution, Harrod.

\section{Introdução}

A moderna teoria do crescimento teve início com a contribuição pioneira de Harrod (1939) em "An Essay in Dynamic Theory", elaborada em maior extensão em seu livro Towards a Dynamic Economics (1948). A sua conclusão

\footnotetext{
1 Doutorando em Economia pela UNICAMP. O autor agradece os comentários de Gilberto Tadeu Lima eximindo-o dos erros remanescentes
} 
principal é que havia uma única taxa de crescimento da economia que validaria as expectativas dos empresários assegurando a sua continuidade (fio da navalha), tendo em vista a propensão a poupar e a relação capital-produto. Esta conclusão perturbou tanto os economistas neoclássicos quanto neokeynesianos, originando, de um lado, a resposta neoclássica através dos modelos de Solow (1956) e Swan (1956) e, por outro, a resposta da escola de Cambridge na mesma época.

O objetivo deste trabalho é discutir o modelo de crescimento de Harrod enquanto motivação para o surgimento dos modelos de crescimento e distribuição pós-keynesianos (do tipo I), basicamente as teorias de Robinson e Kaldor $^{2}$, bem como estudar estes modelos à luz do problema que ficou conhecido como o fio da navalha de Harrod.

Assim, na primeira seção, estudaremos o modelo de Harrod. Nas duas seções seguintes, os modelos de Robinson e Kaldor ${ }^{2}$, respectivamente. Na seção final, faremos uma apresentação concisa dos pontos em comum e divergentes das abordagens destes dois últimos autores.

\section{Modelo de Harrod}

Partindo da usual condição de equilíbrio macroeconômico, temos

$$
I=s Y
$$

na qual $I$ representa o investimento, $Y$ a renda e $s$ uma dada proporção da renda que é poupada. A partir da ótica keynesiana, esta condição significa

\footnotetext{
${ }^{2}$ Estes autores também são rotulados como neokeynesianos. Os modelos de crescimento e distribuição pós-keynesianos atuais (tipo II) são também denominados na literatura como estruturalistas ou modelos à la Kalecki-Steindl e têm como representantes principais Rowthorn (1982), Dutt (1984), Taylor (1985,1991), Bhaduri \& Marglin (1990), entre outros. Uma de suas conclusões principais é que pode haver crescimento com melhoria na distribuição de renda, diferentemente dos modelos do tipo I em que a relação entre crescimento e distribuição é inversa. A relação direta entre crescimento e distribuição em modelos do tipo II se dá através da endogenização do grau de utilização, inclusive no longo prazo. Contudo, para Rowthorn (1982) e Dutt (1984), aumentos no salário real aceleram o crescimento necessariamente, enquanto que, para Bhaduri \& Marglin (1990), esse resultado é uma possibilidade. Para algumas formulações não-lineares recentes na linha desses modelos pós-keynesianos, ver Lima (1998a, 1998b,1999) Ver, em particular, Lima (1998a), em que a utilização de uma função investimento não-linear permite derivar os resultados dos modelos de $1^{\mathrm{a}}$ e $2^{\mathrm{a}}$ geração como casos particulares.
} 
que o investimento gera renda e demanda via efeito multiplicador e o nível de equilíbrio da renda é igual ao volume de investimento multiplicado pelo fator $1 / s$. Dividindo ambos os membros por $k$ (o estoque de capital total da economia) e considerando $v$ (a relação capital-produto) uma constante dada, podemos observar que

$$
g_{w}=\frac{I}{k}=s \frac{Y}{k}=\frac{s}{v}
$$

é a equação "dinâmica" de Harrod. A taxa de crescimento da renda $g_{w}$ é a taxa que compatibiliza o caráter dual do investimento, visto que este gera demanda através do multiplicador e, simultaneamente, cria capacidade produtiva adicional. Essa taxa pode ser interpretada como aquela que valida as expectativas dos capitalistas e, ao validá-las, garante a continuidade do crescimento econô ${ }^{*}$ mico a esta mesma taxa. Esta é denominada como taxa garantida (warranted rate). Sob as condições impostas, $g_{w}$ é um valor único dado pelas constantes $s$ e $v$. Na medida em que a relação capital-produto é uma constante (v), a taxa de crescimento do capital corresponde à taxa de crescimento do produto.

A próxima questão naturalmente é saber se as firmas, no agregado, irão desejar crescer a esta taxa ou, em outros termos, se os planos de investimento das firmas podem ser compatibilizados com uma expansão equivalente à taxa garantida. Harrod $(1948$, p. 86) afirma que não há garantia de que isto possa ocorrer numa economia capitalista, na medida em que as decisões de investimento das firmas baseiam-se em expectativas individuais acerca da taxa de crescimento que seja lucrativa. Assim, não existem motivos para esperar que a taxa garantida seja, de fato, realizada. Se a taxa efetiva $\left(g_{a}\right)$ situar-se abaixo da garantida, a economia se encontrará com capacidade utilizada inferior à planejada e a taxa de crescimento se reduzirá gradualmente. Por outro lado, se a taxa efetiva ficar acima da garantida, o investimento superará a poupança agregada, gerando uma inflação cumulativa. Neste sentido, a trajetória da taxa garantida assemelha-se ao fio da navalha ( $k n i f e$ edge): apenas uma trajetória existe, definida por uma única taxa de crescimento; se a taxa efetiva divergir da taxa garantida, a trajetória efetiva não convergirá. Conforme Harrod (1948, p. 86), "forças centrífugas conjugam-se fazendo com que o sistema se afaste mais e mais da linha de avanço requerida" quando a taxa efetiva for diferente da garantida.

Para que o pleno emprego seja mantido ao longo do tempo, assumindo que ele exista inicialmente, o emprego deverá crescer à taxa de crescimento da força de trabalho. Considere que as taxas de crescimento da produtividade $(\sigma)$ 
e da força de trabalho $(n)$ sejam dadas de forma independente. Assim, a taxa de crescimento natural $\left(g_{n}\right)$ corresponde à seguinte equação

$$
g_{n}=n+\sigma
$$

Desta forma, o crescimento contínuo com pleno emprego requer que

$$
g_{a}=g_{w}=g_{n}
$$

ou seja, que a taxa de crescimento efetivo $\left(g_{a}\right)$ corresponda à taxa garantida e natural. Neste caso, a economia encontra-se em uma idade de ouro (golden age), de acordo com Robinson (1962), o que exige que todas as partes do sistema expandam a uma taxa constante e uniforme igual à taxa natural.

Contudo, se a taxa garantida $g_{w}$ corresponde à relação $s / v$ e a taxa de crescimento natural $g_{n}$ à soma de $n$ com $\sigma$, somente por acidente as duas taxas serão equivalentes, já que seus determinantes são diferentes. Devemos ressaltar, entretanto, conforme Harrod, que, mesmo que uma idade de ouro possa existir, é pouco provável que seja alcançada, pois não há nenhum mecanismo endógeno (inerente) ao sistema capitalista que o conduza ao crescimento com equilíbrio a pleno emprego.

Considerando a inovação técnica e o aumento da força de trabalho como limites independentes à expansão do sistema, bem como a acumulação contínua de capital, de forma a gerar uma poupança equivalente como exigência essencial desta expansão, não existe nenhum mecanismo dentro do sistema que traga estes elementos a uma relação apropriada entre si. Desta maneira, Harrod $(1939$, p. 22) observa o processo de crescimento capitalista como endogenamente instável, ou seja, a economia não mantém um crescimento contínuo de equilíbrio, mas apresenta uma série de booms de investimento seguida por recessões passando por estados de completa estagnação. Neste sentido, em sua visão, a equação "dinâmica" e as relações constitutivas do sistema apresentam a chave para a existência dos ciclos econômicos e períodos de estagnação no capitalismo (Harris, 1978, p. 30).

Ao examinar a base analítica do argumento de Harrod, há várias questões a serem consideradas. No centro do problema, repousa a questão de saber o que governa as decisões de investimento das empresas em uma economia capitalista e se elas, tomadas no agregado, realizarão um volume de investimento à uma taxa compatível com o crescimento contínuo (steady growth).

A unicidade e constância da taxa garantida $\left(g_{w}\right)$ requerem que ambos $s$ e $v$ da equação (2) tenham valores únicos e constantes ao longo do tempo. A taxa de poupança será constante no decorrer do tempo, se as atitudes em relação à poupança e à distribuição de renda entre lucros e salários não se alterarem. 
A constância de $s$ será mantida, se a taxa de juros e a taxa de lucro permanecerem constantes e o progresso técnico for consistente com uma distribuição de renda também constante. Esta última não foi um tópico que recebeu muita atenção de Harrod em seu trabalho. A distribuição de renda foi considerada exógena e, assim, não explicada em seu modelo.

Outro ponto interessante em seu modelo teórico refere-se a como opera o paradoxo da parcimônia. Um valor maior da taxa de poupança $(s)$ em (2) resulta em uma taxa de crescimento de equilíbrio $\left(g_{w}\right)$ maior, ceteris paribus. O paradoxo da poupança de Keynes reflete-se em Harrod $(1948$, p. 88) via taxa efetiva de crescimento $\left(g_{a}\right)$ que pode ser afetada pela relação entre as taxas garantida $\left(g_{w}\right)$ e natural $\left(g_{n}\right)$. Quando $g_{w}$ superar $g_{n}$, há uma tendência da taxa efetiva $\left(g_{a}\right)$ se situar abaixo de $g_{w}$, originando uma recessão através do princípio da instabilidade. É, neste sentido, que uma maior propensão a poupar, ao elevar $g_{w}$ acima de $g_{n}$, torna-se compatível com o paradoxo da poupança (Asimakopulos, 1991, p. 179).

Por outro lado, se a taxa de juros for constante ao longo do tempo e o progresso técnico neutro, então o valor de $v$ em (2) também será constante. De acordo com Harrod, progresso técnico neutro significa que a produtividade do trabalho aumenta a uma mesma taxa em todos os estágios da produção. Em outros termos, Harrod chama de progresso técnico neutro o aumento da produtividade do trabalho sem a modificação de $v$.

O problema da unicidade da trajetória de crescimento contínuo tornouse a parte da teoria de Harrod que despertou maior controvérsia, i. e., por quê existe apenas um único valor para a taxa de crescimento contínuo (taxa garantida $-g_{w}$ ), enquanto que a questão da instabilidade compreendia a própria conclusão keynesiana acerca da inerente falta de estabilidade das economias capitalistas. O fio da navalha implica que uma única trajetória existe, ao passo que a instabilidade concerne à falta de capacidade do sistema convergir ao equilíbrio, se acaso ele estiver fora dele.

Assim, Harrod será atacado, de um lado, por sua falha em lidar explicitamente com a taxa de lucro e distribuição de renda (Robinson e Kaldor) e, por outro, por sua falha em especificar uma relação entre taxa de juros e $v$ consistente com a teoria de produção neoclássica (Solow). A base dessas críticas residia, de ambos lados, na rejeição do fio da navalha, i. e., que havia apenas uma única taxa garantida. Assim, Robinson (1956) e Kaldor (1956) irão flexibilizar a taxa de poupança agregada $(s)$ conforme os vários perfis distributivos e Solow (1956), a relação capital-produto $(v)^{3}$.

\footnotetext{
${ }^{3}$ A suposição de perfeita substitutibilidade entre capital-trabalho permite diversas relações capital-produto $(v)$ tornando múltiplas as taxas garantidas de Harrod O modelo de Solow não será abordado por não ser objeto do presente trabalho.
} 


\section{Modelo de Robinson}

A abordagem de Robinson quanto a teoria do crescimento $(1956,1962)$ envolve dois problemas interligados. O primeiro refere-se ao fio da navalha de Harrod que restringe o crescimento contínuo a uma única taxa (taxa garantida). Na análise robinsoniana, a taxa de poupança agregada é influenciada pela distribuição funcional da renda entre salários e lucros. As poupanças por classe social são exógenas, mas a poupança agregada da economia é endógena, variando com a distribuição de renda. Neste sentido, um dos elementos básicos de qualquer análise de crescimento econômico deve ser uma teoria de distribuição de renda. A rejeição da abordagem neoclássica da distribuição ${ }^{4}$ requer a formulação de uma teoria que determine a taxa de lucro ou o salário real.

O fato de que não havia nenhuma teoria de lucro neoclássica logicamente coerente, a fez voltar ao princípio da demanda efetiva para uma explicação dos lucros. Neste sentido, a sua dívida intelectual com Kalecki tenha sido talvez mais importante do que em relação a Keynes da Teoria Geral (1936). Da mesma forma que Kaldor (1956), ela fez uso do mecanismo do multiplicador para derivar a relação entre a taxa de crescimento econômico e a taxa de lucro.

Partindo da conhecida identidade kaleckiana

$$
P+W=C_{c}+C_{w}+I
$$

na qual $P=$ lucros, $W=$ salários, $C_{c}=$ consumo dos capitalistas, $C_{w}=$ consumo dos trabalhadores e $I=$ investimento, e assumindo que os trabalhadores não poupam, temos que

$$
P=C_{c}+I
$$

ou seja, os lucros são determinados pelas decisões de gasto dos capitalistas. Se $C_{c}=0$,

$$
P=I
$$

que dividido por $k$ (estoque de capital), encontramos

$$
r=g
$$

\footnotetext{
${ }^{4}$ A teoria neoclássica utiliza a função de produção agregada para encontrar os preços relativos dos 'fatores' capital e trabalho. Mas, esta abordagem, cf. Robinson, envolve um raciocínio circular, pois se salários e lucros entram nos preços dos bens de capital, então ao agregar capital em termos nominais para obter a taxa de lucro via produtividade marginal do capital, implica conhecer a taxa de lucro antes de encontrar os preços compatíveis com esta taxa, segundo Kregel (1972).
} 
i. e., a taxa de lucro $r=P / k$ é determinada pela taxa de acumulação $g=I / k$. Neste específico caso, assumimos uma condição restritiva de que a propensão a poupar dos capitalistas $(s)$ é igual a $1\left(C_{c}=0\right)$. De outra forma, podemos observar que

$$
I=S=s P \quad(9), 0<s \leq 1 .
$$

Dividindo ambos os lados por $k$, obtemos a famosa equação de Cambridge na qual

$$
g=s r
$$

A relação entre lucros e investimento, que figura proeminentemente na teoria do ciclo econômico de Kalecki, é baseada na influência das condições atuais sobre as expectativas atuais de longo prazo. Os investimentos atuais (determinados por decisões feitas em períodos anteriores baseados em lucros anteriores) têm um efeito positivo sobre os lucros atuais, enquanto estes lucros têm um efeito positivo sobre as decisões de investimento atuais e também sobre os gastos de investimento futuro. Ao levar em conta o valor do estoque de capital, a relação anterior, que era entre lucro e investimento, transforma-se agora entre taxa de lucro e taxa de acumulação. Este é um ponto importante, pois permite à autora derivar a taxa desejada de acumulação. Esta é a taxa que resulta numa taxa de lucro que induz esta particular taxa de acumulação. Em outros termos, a taxa desejada é aquela que faz as firmas se sentirem satisfeitas com a situação na qual se encontram. É uma taxa de equilíbrio estável.

Robinson distingue entre a taxa desejada e taxas possíveis de crescimento, uma distinção comparável àquela feita por Harrod entre a taxa garantida e natural. A taxa desejada pode ser insuficiente para resultar em pleno emprego, tendo em vista a taxa de crescimento da população e de produtividade. Quando a taxa desejada tende a exceder a taxa de crescimento compatível com o crescimento da força de trabalho, Robinson observa que os "espíritos animais" podem obter êxito em aumentar a taxa de progresso técnico o suficiente para igualar a taxa possível à desejada. Contudo, se a taxa desejada for tão alta que a taxa de crescimento da produtividade não conseguir acompanhá-la, então a taxa efetiva terá que ser reduzida.

A taxa desejada de acumulação é análoga à taxa garantida de Harrod. Entretanto, a primeira baseia-se na interação entre a acumulação e distribuição que é ausente na teoria de Harrod. A taxa garantida depende diretamente da propensão a poupar e da natureza da tecnologia.

O paradoxo da poupança de Keynes aparece na teoria de Robinson como uma relação inversa entre o grau de parcimônia e a taxa desejada de acumulação. Assim, quando a taxa efetiva de acumulação for limitada apenas pela taxa desejada, quanto maior o grau de parcimônia, menor a taxa de acumulação, 
diferentemente da taxa garantida de Harrod que está diretamente relacionada com a propensão a poupar da economia, como observado acima.

E assim, Robinson conclui que, para uma dada taxa agregada de poupança $s$, uma maior taxa de acumulação define uma maior taxa de lucro de equilíbrio e um salário real menor, produzindo uma distribuição de renda que iguala a poupança agregada $(S)$ ao investimento $(I)$. Neste sentido, diferentes taxas de investimento em relação ao produto estarão associadas com diferentes participações de lucro e salário na renda nacional, de forma que o valor de s mudará com a distribuição de renda e a taxa de poupança será livre para se adaptar a qualquer dada taxa de acumulação, incluindo a taxa natural $\left(g_{n}\right)$ determinada pelo progresso técnico e crescimento populacional. Desta maneira, não havia uma única taxa garantida. Haveria tantas $g_{w}$ quantas fossem as distribuições de renda.

A variável fundamental em seu modelo teórico é o investimento determinado pelos "espíritos animais". Em outras palavras, a acumulação de capital é determinada pela expectativa da taxa de lucro e é ela (a acumulação de capital) que determina a taxa de lucro efetiva. Há limites para o valor que a taxa de investimento pode assumir ao longo do tempo. O limite superior é definido pela barreira inflacionária, que resulta num salário real tão baixo que os trabalhadores reagem, elevando os salários nominais, causando maiores taxas inflacionárias. De forma contrária, existe uma taxa de investimento que é tão baixa que a taxa de lucro correspondente não é suficiente para justificar o investimento realizado, o que causa reduções no investimento seguinte e recessão. Dentro destes limites, crescimento estável de equilíbrio é possível, inclusive à taxa natural, se os capitalistas se dispuserem a realizar os investimentos necessários a esta taxa.

Apesar de sua dívida intelectual em relação a Kalecki, o trabalho de Robinson sobre o crescimento das economias capitalistas pode ser visto como uma extensão da Teoria Geral de Keynes para o longo prazo. A taxa de investimento é independente da propensão a poupar, com o nível de poupança se acomodando a um volume de investimento determinado pelas firmas. Uma parte importante desta extensão é a teoria da distribuição de renda de Kalecki e a dupla relação de Kalecki entre investimento e lucro, a qual Robinson transforma entre a taxa de acumulação e a taxa de lucro. A taxa atual de acumulação é um determinante da taxa atual de lucro, ao passo que o último é um determinante das decisões atuais de investimento e da futura taxa de acumulação. Esta dupla relação fornece os elementos para sua definição da taxa desejada de acumulação, sendo análoga à taxa garantida de Harrod. Uma importante diferença entre elas é que Robinson faz uso direto da indução a investir e o 
efeito da propensão a poupar, dado o investimento, sobre a distribuição de renda. Como resultado, o paradoxo da parcimônia de Keynes aparece na teoria de Robinson como uma relação inversa entre o grau de poupança e a taxa desejada de acumulação.

Ao definir a taxa desejada, Robinson assume que as expectativas são formadas de tal forma - a projeção dos níveis atuais de lucro para o futuro - que a taxa desejada é estável. Há o reconhecimento, contudo, que as expectativas poderiam ser formadas de forma diferente pela projeção de mudanças recentes no nível dos lucros, então o modelo seria inerentemente instável.

A teoria de acumulação de Robinson pode ser entendida como fiel à visão de Keynes acerca da operação das economias capitalistas. O presente representa um breve intervalo de tempo entre um passado irrevogável e um futuro desconhecido. Em tal cenário, a determinação do investimento não pode ser reduzida a alguma fórmula que pode ser repetida ao longo do tempo. O modelo de crescimento de Robinson é, assim, aberto. Ele fornece um aparato para examinar importantes elementos que afetam a taxa de acumulação ao longo do tempo, mas seus valores para estes elementos dependem de fatores históricos, institucionais e psicológicos.

\section{Modelo de Kaldor}

O mesmo mecanismo keynesiano de distribuição de renda está por trás do modelo de crescimento de Kaldor (1956). A sua explicação, tal como em Robinson, baseia-se na hipótese básica que o investimento é a variável autônoma efetiva do sistema. A poupança agregada em relação à renda $(s)$ não pode ser considerada uma constante, pois é uma média ponderada das propensões a poupar dos capitalistas e trabalhadores.

Sendo $S_{w}$ e $S_{p}$ as poupanças agregadas a partir de salários e lucros, respectivamente, podemos escrever o seguinte conjunto de identidades:

$$
\begin{aligned}
& Y \equiv W+P \\
& I \equiv S \\
& S \equiv S_{w}+S_{p}
\end{aligned}
$$

Expressando as funções de poupança como $S_{w}=s_{w} W$ e $S_{p}=s_{p} P$, encontramos $I=s_{p} P+s_{w} W=s_{p} P+s_{w}(Y-P)=\left(s_{p}-s_{w}\right) P+s_{w} Y$, de onde resulta:

$$
\frac{I}{Y}=\left(s_{p}-s_{w}\right) \frac{P}{Y}+s_{w}
$$


Rearranjando os termos, obtemos

$$
\frac{P}{Y}=\frac{1}{s_{p}-s_{w}} \frac{I}{Y}-\frac{s_{w}}{s_{p}-s_{w}}
$$

que é a equação fundamental do modelo de Kaldor. É essencial para a compreensão da teoria distributiva de Kaldor que consideremos $P / Y$, a distribuição de renda, como variável dependente e $I / Y$, a taxa de investimento em relação ao produto, como variável independente. Esta indicação de causalidade indica a natureza keynesiana da teoria de Kaldor.

Fazendo uma pequena modificação na equação fundamental, podemos obter

$$
\frac{P}{k}=\frac{1}{s_{p}-s_{w}} \frac{I}{k}-\frac{s_{w}}{s_{p}-s_{w}} \frac{Y}{k}
$$

A equação (11) significa que existe uma distribuição de renda entre salários e lucros e uma correspondente taxa de lucro - equação (12) - à qual a condição de equilíbrio permanece satisfeita. Dois casos particulares de especial interesse surgem quando $s_{w}=0$, assim (11) e (12) transformam-se em

$$
\frac{P}{Y}=\frac{1}{s_{p}} \frac{I}{Y} \quad \text { e } \frac{P}{k}=\frac{1}{s_{p}} \frac{I}{k} \text { (esta é a equação de Cambridge) e }
$$

quando $S_{p}=1$ (além de $s_{w}=0$ ), então temos

$$
\frac{P}{Y}=\frac{I}{Y} \mathrm{e} \frac{P}{k}=\frac{I}{k}
$$

Desta forma, a resposta que Kaldor dava a Harrod era muito clara, qual seja, haveria múltiplas taxas garantidas na medida em que haveria múltiplos perfis distributivos e, portanto, diversas taxas agregadas de poupança $(s)$. Em outros termos,

$$
s=\frac{S}{Y}=s_{w} \frac{W}{Y}+s_{p} \frac{P}{Y},
$$

em que $s_{w}$ e $s_{p}$ funcionam como "pesos" na média ponderada $s$.

A teoria de distribuição de renda de Kaldor foi rotulada pelo próprio autor como keynesiana porque retomava algumas idéias básicas de Keynes em sua obra $A$ Treatise on Money (1930). De fato, em uma passagem que se tornaria conhecida na literatura econômica como widow's cruse (jarro da viúva), 
Keynes sugere que o rendimento dos empresários é o resultado de suas decisões de gastos, e não o contrário. Mais especificamente, como enfatiza Kaldor, a equação (11) é uma extensão, para um dado nível de renda, do princípio do multiplicador keynesiano. Na Teoria Geral, o mecanismo do multiplicador referia-se à determinação do nível de emprego e produto, a partir das decisões de gastos, para uma dada distribuição de renda; no Treatise, conforme Kaldor, o princípio do multiplicador refere-se à determinação da relação entre preços e salários (e, portanto, da distribuição de renda), a partir das decisões de gastos, para um dado nível de produto. De forma simplificada, o mecanismo do multiplicador desencadeado por um desequilíbrio entre poupança e investimento levará a uma alteração no nível geral de preços. Esta modificação gerará lucros ou perdas inesperadas, configurando uma nova distribuição de renda entre lucros e salários.

Como condição de estabilidade do modelo, temos que

$$
\begin{aligned}
& s_{p}>s_{w} \text { ou } \\
& s_{w}<\frac{I}{Y} \quad \text { (13) e } s_{p}>\frac{I}{Y}
\end{aligned}
$$

A condição (13) exclui o caso de um equilíbrio dinâmico com uma participação do lucro na renda negativa ou nula, e a restrição (14) exclui o equilíbrio dinâmico com participação dos salários na renda negativa ou nula. $\mathrm{Na}$ prática, se a condição (13) não for atendida, o sistema entrará em uma situação de subemprego crônico. Por outro lado, se a condição (14) não for satisfeita, o sistema entraria numa situação de inflação crônica. Dentro destes limites, as equações (11) e (12) mostram a existência de uma distribuição de renda e taxa de lucro que, ao longo do tempo, manterá o sistema em equilíbrio.

Em todo seu trabalho sobre crescimento, Kaldor (1959, p.296) assume que o equilíbrio estável no longo prazo implica pleno emprego. Seu argumento, de forma simples, é que, se o pleno emprego não fosse mantido, não haveria possibilidade de um equilíbrio contínuo. Portanto, a análise de crescimento contínuo de longo prazo poderia começar assumindo o pleno emprego.

Em seu trabalho, os empresários decidem olhando para o futuro. $\mathrm{O}$ aspecto crucial desses empresários é seu dinamismo técnico: é sua atitude básica direcionada à expansão que gerará progresso técnico e acumulação de capital. O canal de transmissão é direto: suficiente dinamismo causará a introdução de progresso técnico na forma de novas máquinas, que Kaldor pensa como "o principal motor do crescimento econômico" (1962, p. 188). 


\section{Conclusão}

A teoria neokeynesiana ou de Cambridge traz para a linha de frente de análise o motor expansionista das firmas e seu dinamismo técnico como forças básicas que governam o sistema capítàlista. A teoria focaliza as interconexões entre lucro e acumulação através das quais estas forças operam. A taxa de lucro emerge da análise como determinada dentro das condições de poupança e consumo dos diversos grupos de renda consistente com a taxa de acumulação. É evidente que não há lugar nesta teoria para observar a taxa de lucro relacionada com a produtividade do capital visto como um input técnico na produção.

Dentro desta concepção, a ênfase é dada na existência do desemprego e excesso de capacidade enquanto características crônicas de operação da economia capitalista, o que, por sua vez, está intimamente relacionadas com o volume de investimento realizado de um período ao outro ${ }^{5}$. Não há nenhum mecanismo automático de ajustamento capaz de levar a economia ao pleno emprego. Neste respeito, Kaldor é exceção ${ }^{6}$.

Nesta teoria, o principal obstáculo para se alcançar a idade de ouro não advém necessariamente do lado da poupança, dos preços, da distribuição ou da tecnologia. Estes não são vistos como fontes básicas de contradição. A origem é para ser encontrada nas decisões que governam a acumulação: os "espíritos animais" dos capitalistas (Robinson) ou o dinamismo técnico da economia capitalista (Kaldor). Segundo Robinson, o que faz a acumulação de capital ser alta ou baixa depende das características históricas, políticas e psicológicas da economia. A este conjunto de fatores, ela denominou de "espíritos animais", termo este originado de Keynes que o usou com referência principalmente à incerteza envolvida nas decisões de investimento. Para Kaldor, dinamismo

\footnotetext{
${ }^{5}$ Ainda que Robinson $(1956,1962)$ reconheça esta característica, implicitamente ela parece supor que este excesso de capacidade tende ao normal ou planejado no longo prazo. Com isso, apenas ajustes de preços são supostos como operantes. Daí, a relação necessariamente inversa entre crescimento (taxa de lucro) e distribuição (salário real). A relação direta entre crescimento e distribuição em modelos mais recentes se dá via endogenização do grau de utilização, inclusive no longo prazo, como vimos na nota 1

${ }^{6}$ Para Kaldor, no equilibrio de longo prazo, tem-se o pleno emprego. Neste aspecto, sua análise difere radicalmente de Robinson. Kaldor justifica esta conclusão, entre outros motivos, porque assume uma específica função investimento que a faz depender do nível da renda e que mudanças na renda geram uma maior variação no investimento que na poupança. Uma das críticas que se pode fazer a este argumento é que, se variações no produto forem permitidas no curto prazo, o pleno emprego se dará inclusive no curto prazo, conclusão essa que o próprio Kaldor rejeitaria, cf. Dutt (1990)
} 
técnico significava a inventividade e a facilidade à mudança ou ao experimento. Era este dinamismo que, em sua visão, era responsável por taxas altas ou baixas de acumulação e de produto.

Neste sentido, seria possível obter, dentro dos limites de viabilidade técnica, um estado de crescimento de equilíbrio a qualquer taxa concebível, dependendo apenas dos "espíritos animais" ou da extensão do dinamismo técnico. Dito de outra forma, esta teoria revela que poderiam existir múltiplas taxas garantidas.

A resposta que a escola de Cambridge deu ao fio da navalha de Harrod foi no sentido de reconhecer que há diferentes taxas de poupança associadas a diferentes classes sociais. A taxa de poupança agregada, assim, depende da distribuição de renda entre as classes sociais. Há uma série de taxas de lucro consistentes com dada tecnologia e um amplo leque de taxas garantidas. $\mathrm{O}$ problema da unicidade da taxa garantida, assim, desaparece. A existência de uma idade de ouro pode ser vista, então, como o problema de se encontrar a distribuição de renda apropriada: aquela específica distribuição que, para dadas as taxas de poupança das classes sociais, origina uma taxa agregada de poupança tal que a taxa garantida seja igual à taxa natural, supondo que a taxa de acumulação seja a necessária para tanto.

\section{Referências bibliográficas}

ASIMAKOPULOS, A. (1991), Keynes's General Theory and Accumulation, Cambridge University Press.

BHADURI, A. e MARGLIN, S. (1990), "Unemployment and the Real Wage: the Economic Basis for Contesting Political Ideologies", Cambridge Journal of Economics, 14.

DUTT, A. K. (1984), "Stagnation, Income Distribution and Monopoly Power", Cambrige Journal of Economics, 8.

(1990), Growth, Distribution, and Uneven Development, Cambridge University Press.

HARRIS, D. J. (1978), Capital Accumulation and Income Distribution, Stanford University Press.

HARROD, R. F. (1939), "An Essay in Dynamic Theory" in Stiglitz, J. E. e Uzawa, H. (1969), Readings in the Modern Theory of Economic Growth, The MIT Press.

(1948), Towards a Dynamic Economics, Macmillan. 
KALDOR, N. (1956), “Alternative Theories of Distribution", in Stiglitz, J. E. e Uzawa, H. (1969), Readings in the Modern Theory of Economic Growth, The MIT Press.

(1959), "Economic Growth and the Problem of Inflation", partes I e II, Econômica, XXVI, Ago-Nov.

e Mirrlees, J. A. (1962), "A New Model of Economic Growth" in

Stiglitz, J. E. e Uzawa, H. (1969), Readings in the Modern Theory of Economic Growth, The MIT Press.

KEYNES, J. M. (1930), A Treatise on Money, Macmillan.

(1936), The General Theory of Employment, Interest and Money, Macmillan.

KREGEL, J. A. (1972), The Theory of Economic Growth, Macmillan.

LIMA, G. T. (1998a), "A Non-Linear Dynamics of Capital Accumulation, Distribution and Conflict Inflation", in Anais do $26^{\circ}$. Encontro Nacional de Economia, ANPEC, Vitória, Dezembro.

(1998b), "Endogenous Technological Innovation, Capital Accumulation and Distributional Dynamics", Texto para Discussão, 98, CPGE/UFRGS, Porto Alegre.

(1999), "Market Concentration and Technological Innovation in a Macrodynamics of Growth and Distribution" in Anais do $27^{\circ}$. Encontro Nacional de Economia, ANPEC, Belém, Dezembro.

ROBINSON, J. (1956), The Accumulation of Capital, Macmillan.

(1962), Essays in the Theory of Economic Growth, Macmillan.

ROWTHORN, R. (1982), "Demand, Real Wages, and Economic Growth", in Sawyer, M. C. (1988), Post-Keynesian Economics, Edward Elgar.

SOLOW, R. M. (1956), "A Contribution to the Theory of Economic Growth", in Stiglitz, J. E. e Uzawa, H. (1969), Readings in the Modern Theory of Economic Growth, The MIT Press.

SWAN, T. W. (1956), "Economic Growth and Capital Accumulation", in Stiglitz, J. E. e Uzawa, H. (1969), Readings in the Modern Theory of Economic Growth, The MIT Press.

TAYLOR, L. (1985), "A Stagnationist Model of Economic Growth", Cambridge Journal of Economics, 9.

(1991), Income Distribution, Inflation, and Growth - Lectures on Structuralist Macroeconomic Theory, The MIT Press. 